\title{
Aplicación móvil para la contratación de servicios profesionales
}

Fecha de recepción:2021-07-19 • Fecha de aceptación: 2021-09-03 • Fecha de publicación: 2021-10-10

\author{
Henry Daniel Rojas Cisneros \\ Biometrika Cloud Services, Ecuador \\ henry.red1@hotmail.com \\ https://orcid.org/0000-0001-8157-760X
}

\section{RESUMEN}

El presente trabajo se enfoca en la automatización mediante una aplicación móvil y una página administrativa, de la contratación de servicios profesionales. Esta idea surge de la crisis sanitaria generada por el COVID-19, que afectó principalmente a los aspectos sociales y económicos, lo que provocó un incremento en el indicador de desempleo en la mayoría de los países. Durante esta crisis, empresas que ofrecen sus servicios enfocados en entregas a domicilio, mediante aplicaciones móviles, tuvieron un gran crecimiento debido a su gran demanda por la situación actual; sin embargo, otro tipo de servicios, como los de profesionales informales, se vieron gravemente afectados al no contar con ninguna aplicación que facilite su contratación. Para el desarrollo de este proyecto se utilizó la herramienta de desarrollo Android Studio con el lenguaje de programación Kotlin para la aplicación móvil, y para la página administrativa el Framework Angular, sobre el lenguaje Typescript, ambas aplicaciones se conectan con la plataforma Firebase, que permite la gestión de la información y evita la gestión de un servidor, lo que acelera el desarrollo del lado del cliente.

\section{PALABRAS CLAVE: kotlin, typescript, framework, covid.}

\section{ABSTRACT}

The present work focuses on the automation through a mobile application and an administrative page, of the contracting of professional services. This idea arises from the health crisis generated 
by COVID-19, which affected mainly social and economic aspects, which caused an increase in the unemployment indicator in most countries. During this crisis, companies offering their services focused on home deliveries, through mobile applications, had a great growth due to their high demand because of the current situation; however, other types of services, such as informal professionals, were severely affected by not having any application to facilitate their recruitment. For the development of this project, the Android Studio development tool was used with the Kotlin programming language for the mobile application, and for the administrative page the Angular Framework, on Typescript language, both applications are connected to the Firebase platform, which allows the management of information and avoids the management of a server, which speeds up the development of the client side.

KEYWORDS: kotlin, typescript, framework, covid. 


\section{Introducción}

El mundo entero está viviendo una pandemia global por consecuencia del COVID-19, lo que ocasionó una crisis económica en la mayoría de países y que afectó a diferentes aspectos, tanto social, como económico. El indicador que resaltó por su incremento fue el desempleo, el cual afecta directamente al núcleo familiar (Lozano Chaguay et al., 2020). Sin embargo, de acuerdo con la OIT (2021), el número de plataformas digitales de trabajo en la región ha aumentado 14 veces, haciendo que el COVID-19 acelere las transformaciones. Por lo que surge la necesidad de aprovechar mejor las oportunidades de empleo y negocios, en un sector donde predomina la gestión por algoritmos.

En Ecuador, como en muchos países de la región latinoamericana, las y los trabajadores informales han sido seriamente perjudicados al estar más expuestos al contagio por la naturaleza de su trabajo (Grijalva, 2020). Empresas como Uber (s.f.), Cabify (s.f.), InDriver (s.f.), etc., dedicadas al servicio de transporte y reparto, han crecido debido a la incorporación de trabajadores informales o que perdieron su empleo. Sin embargo, otro tipo trabajadores que ofrecen servicios como electricista, plomero, albañil, sastre, carpintero, entre otros, no cuentan con un medio tecnológico que ofrezca un servicio de similares características, el cual facilite encontrar una oportunidad de trabajo.

Aunque existan portales que permiten la publicación de ofertas laborales como OpciónEmpleo (s. f.), CompuTrabajo (s. f.), Multitrabajos (s. f.), entre otros, estos servicios se enfocan en ofrecer una bolsa de empleos o postulación con el envío del curriculum vitae a la oferta publicada, proceso que puede tardar o no tener ninguna respuesta.

Por lo mencionado anteriormente, el siguiente trabajo tiene como objetivo el desarrollo de una aplicación móvil prototipo que permita mejorar el proceso de contratación de servicios profesionales de cualquier área, con la publicación de ofertas laborales instantáneas de los usuarios que la utilicen, lo que facilitará la comunicación y llegar a un acuerdo por el trabajo solicitado, para su cumplimento, se estableció lo siguiente: definir los requerimientos y restricciones de la aplicación, desarrollar la aplicación utilizando los estándares y metodología establecidos, y validar el funcionamiento de la aplicación.

\section{Metodología}

Se inició con la definición del proceso de contratación actual, en la Figura 1 se muestran los pasos que siguen los solicitantes para la contratación de servicios profesionales desde la búsqueda, consulta de disponibilidad, acuerdo verbal y culminación. 


\section{Figura 1}

Proceso actual - Sin automatizar

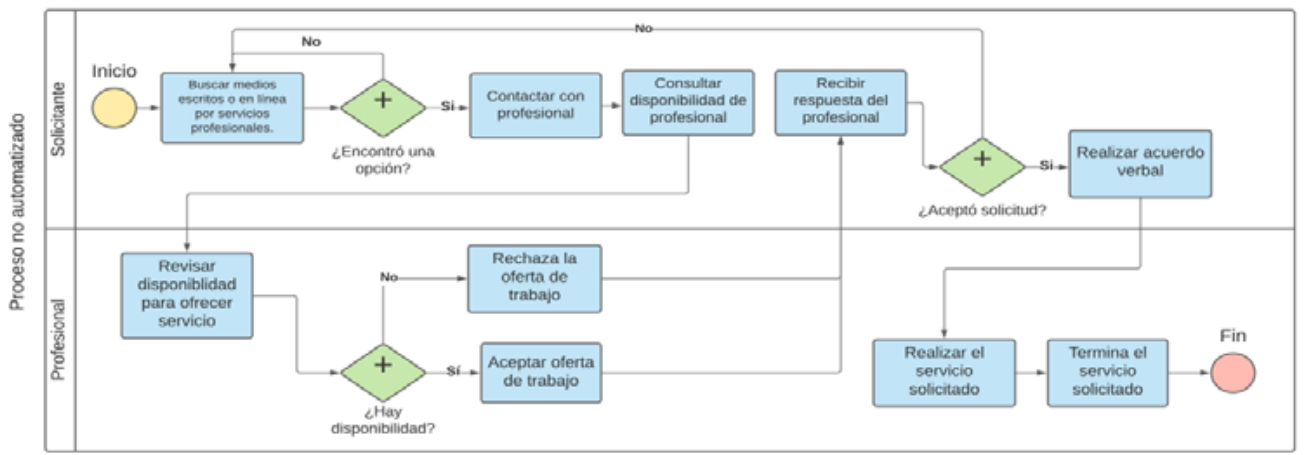

A continuación, se define el proceso automatizado, en la Figura 2 se observa cómo se va a automatizar el proceso con el uso de la aplicación móvil, tomando en cuenta a cada involucrado dentro de este proceso.

Figura 2

Diagrama proceso automatizado

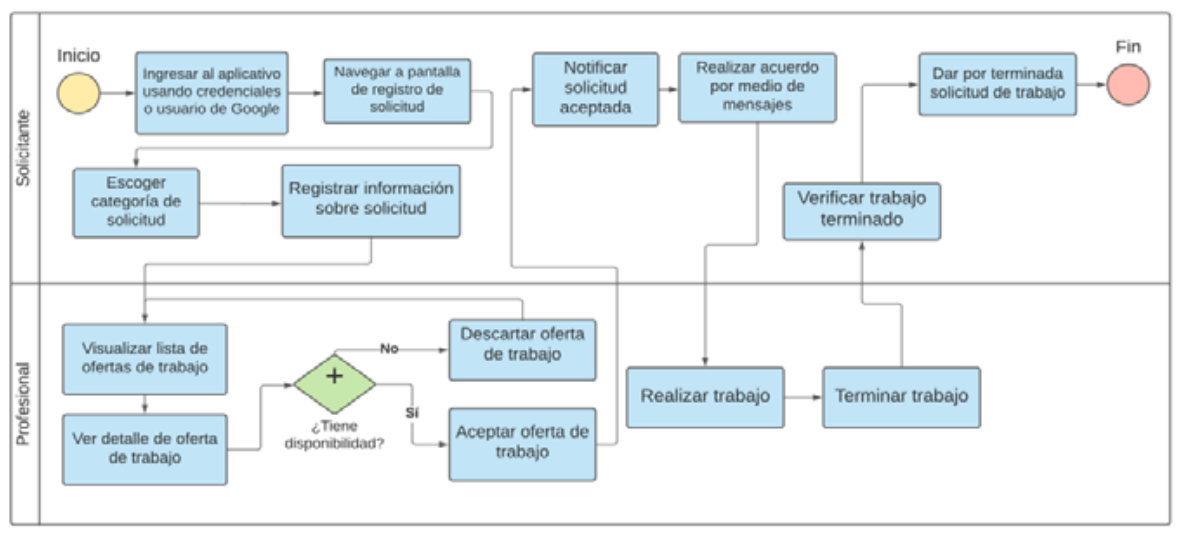

\subsection{Especificación de requerimientos}

En base a la definición de los procesos se definen los requisitos funcionales para la aplicación móvil y la página administrativa, estas funcionalidades se las describe en detalle en las historias de usuario.

Se establecieron las siguientes funciones base que tendrá la aplicación móvil, estas se enlistan a continuación: 
- Iniciar sesión y registro de usuarios.

- Publicar y visualizar ofertas de trabajo por categoría.

- Cambiar estado de solicitudes de trabajo.

- Calificar solicitud de trabajo terminada.

- Visualizar histórico de ofertas de trabajo.

- Visualizar perfil de usuario.

- Enviar comentarios en solicitud de trabajo aceptada.

- Notificar solitud de trabajo aceptada y envío de comentarios.

Se establecieron las siguientes funciones base que tendrá la plataforma web, estas se enlistan a continuación:

- Iniciar sesión en página administrativa para usuario único

- Visualizar y deshabilitar usuarios del sistema

- Visualizar ofertas de trabajo por categorías.

- Registrar, editar, deshabilitar y visualizar categorías.

En la Tabla 1 se muestra un ejemplo de historia de usuario definido para la publicación de ofertas de trabajo para el usuario solicitante.

\section{Tabla 1}

Historias de usuario publicar oferta de trabajo

\begin{tabular}{lc}
\hline Húmero: 3 & $\begin{array}{l}\text { Nombre: Publicar oferta de } \\
\text { trabajo }\end{array}$ \\
\hline Usuario: Solicitante/Cliente & Riesgo en Desarrollo: Alta \\
\hline Prioridad en negocio: Alta & Iteración asignada: 2 \\
\hline $\begin{array}{l}\text { Descripción: los solicitantes deberán escoger una categoría a la cual } \\
\text { pertenecerá la oferta de trabajo a publicar, y de acuerdo con la categoría, } \\
\text { aparecerán los campos del formulario que deben ser llenados de forma } \\
\text { obligatoria. }\end{array}$ \\
\hline $\begin{array}{l}\text { Observación: cada categoría tiene sus campos específicos a mostrar, se } \\
\text { limita a un máximo de } 5 \text { fotografías para respaldo fotográfico, en caso de que } \\
\text { los datos ingresados sean incorrectos se mostrarán mensajes indicando los } \\
\text { errores antes de enviar la información }\end{array}$ \\
\hline
\end{tabular}




\subsection{Restricciones}

Considerando que el proyecto tuvo como principal objetivo el desarrollo de un prototipo funcional, se establecieron las siguientes restricciones.

Para la aplicación móvil:

- El desarrollo de la aplicación móvil se lo realizará en el IDE de desarrollo Android Studio (Developers, 2021) con el lenguaje de programación Kotlin, que toma lo mejor de JAVA y se enfoca en la productividad.

- La aplicación móvil no utilizará ningún proceso relacionado con el cobro del servicio prestado por el profesional.

- El dispositivo que utilice la aplicación debe tener conexión a Internet para iniciar sesión y tener acceso a la información almacenada en Firebase.

- La aplicación móvil será compatible desde la versión 5.0 o posterior.

- No se realizará la publicación de la aplicación en la tienda virtual de aplicaciones Google Play.

Para la plataforma web:

- Se utilizará Angular como Framework de desarrollo, con el lenguaje de programación Typescript y como IDE de desarrollo Visual Code (Aitana Soluciones ERP y CRM, 2018) para el desarrollo de la funcionalidad.

- El backend de la plataforma usará Firebase para la persistencia de la información y la gestión de usuarios.

- Contar con conexión a Internet para el acceso a la información almacenada en Firebase.

- Se tendrán registradas 10 categorías iniciales, esto debido a que el presente proyecto únicamente es un prototipo.

En el desarrollo del proyecto se utilizó la metodología de desarrollo ágil Scrum, un marco de trabajo iterativo incremental que estructura el desarrollo en ciclos de trabajo llamados sprints. Scrum pone énfasis en productos que funcionen al final de cada sprint, en el caso del software que su código esté integrado, completamente probado y listo para entregar (Deemer et al., 2009).

A continuación, se muestran cada uno de los roles, artefactos y eventos principales que se llevaron a cabo para el presente proyecto.

En la Tabla 2 se muestran los roles que tienen los diferentes encargados de la gestión del proyecto. 
Tabla 2

Equipo de trabajo y roles

\begin{tabular}{ll}
\hline \multicolumn{1}{c}{ Personas / Entidad } & \multicolumn{1}{c}{ Rol } \\
\hline Universidad Tecnológica Israel & Product Owner \\
\hline Mg. Renato Toasa & Scrum Master \\
\hline Henry Rojas & Development Team \\
\hline
\end{tabular}

En la Tabla 3 muestra el product backlog del proyecto, que enlista todas las funcionalidades a desarrollarse, el sprint al que pertenecen y el estado.

Tabla 3

Product Backlog

\begin{tabular}{|c|c|c|c|}
\hline N. Historia & Enunciado product backlog & Sprint & Estado \\
\hline 1 & Iniciar sesión usuarios finales & 1 & Terminado \\
\hline 2 & Registro de usuarios finales & 1 & Terminado \\
\hline 3 & Publicar oferta de trabajo & 2 & Terminado \\
\hline 4 & Visualizar ofertas de trabajo publicadas & 3 & Terminado \\
\hline 5 & Finalizar solicitud de trabajo & 4 & Terminado \\
\hline 6 & Calificar solicitud de trabajo terminado & 4 & Terminado \\
\hline 7 & Visualizar histórico de ofertas de trabajo & 3 & Terminado \\
\hline 8 & Visualizar ofertas publicadas por categoría & 3 & Terminado \\
\hline 9 & Visualizar detalle de oferta de trabajo & 3 & Terminado \\
\hline 10 & Aceptar oferta de trabajo & 4 & Terminado \\
\hline 11 & Terminar solicitud de trabajo & 4 & Terminado \\
\hline 12 & Visualizar perfil de usuario & 5 & Terminado \\
\hline 13 & Notificar solitud de trabajo aceptada & 6 & Terminado \\
\hline 14 & Notificar envío de comentarios & 6 & Terminado \\
\hline 15 & Enviar comentarios en solicitud de trabajo aceptada & 5 & Terminado \\
\hline 16 & Iniciar sesión en página administrativa & 1 & Terminado \\
\hline 17 & Visualizar usuarios del sistema & 2 & Terminado \\
\hline 18 & Deshabilitar usuarios del sistema & 2 & Terminado \\
\hline 19 & Visualizar ofertas de trabajo por categorías & 2 & Terminado \\
\hline 20 & Registrar categoría & 1 & Terminado \\
\hline 21 & Editar categoría & 1 & Terminado \\
\hline 22 & Deshabilitar categorías & 1 & Terminado \\
\hline 23 & Visualizar categorías registradas & 1 & Terminado \\
\hline
\end{tabular}


En la Tabla 4 se observa uno de los sprint backlog del proyecto, que enlista todas las tareas que se realizaron para cumplir con los enunciados propuestos en el product backlog, con su tiempo estimado de desarrollo.

\section{Tabla 4}

\section{Sprint Backlog 2}

\begin{tabular}{|c|c|c|c|}
\hline $\begin{array}{l}\text { Identificador } \\
\text { product backlog }\end{array}$ & $\begin{array}{l}\text { Enunciado product } \\
\text { backlog }\end{array}$ & Tareas / Actividades & $\begin{array}{l}\text { Tiempo estimado } \\
\text { (Horas) }\end{array}$ \\
\hline \multirow[t]{14}{*}{2} & \multirow[t]{5}{*}{$\begin{array}{l}\text { Publicar oferta de } \\
\text { trabajo }\end{array}$} & $\begin{array}{l}\text { Creación de navegabilidad y } \\
\text { visualización de pantallas por tipo de } \\
\text { usuario. }\end{array}$ & \multirow[t]{5}{*}{20} \\
\hline & & $\begin{array}{l}\text { Diseño de interfaz para publicación de } \\
\text { ofertas de trabajo. }\end{array}$ & \\
\hline & & $\begin{array}{l}\text { Creación de funcionalidad para } \\
\text { formulario y conexión con Firebase. }\end{array}$ & \\
\hline & & Validaciones para campos de interfaz. & \\
\hline & & $\begin{array}{l}\text { Pruebas de funcionalidad } \\
\text { implementada. }\end{array}$ & \\
\hline & \multirow[t]{3}{*}{$\begin{array}{l}\text { Visualizar usuarios } \\
\text { del sistema }\end{array}$} & $\begin{array}{l}\text { Diseño de interfaz para visualización } \\
\text { de usuarios registrados. }\end{array}$ & \multirow[t]{3}{*}{20} \\
\hline & & $\begin{array}{l}\text { Creación de funcionalidad para } \\
\text { conexión con Firebase y obtención de } \\
\text { usuarios registrados. }\end{array}$ & \\
\hline & & $\begin{array}{l}\text { Pruebas de funcionalidad } \\
\text { implementada. }\end{array}$ & \\
\hline & \multirow{3}{*}{$\begin{array}{l}\text { Deshabilitar } \\
\text { usuarios del } \\
\text { sistema }\end{array}$} & $\begin{array}{l}\text { Implementación de botón deshabilitar } \\
\text { en ítems de usuarios. }\end{array}$ & \multirow[t]{3}{*}{5} \\
\hline & & $\begin{array}{l}\text { Creación de funcionalidad para botón } \\
\text { deshabilitar y conexión con Firebase. }\end{array}$ & \\
\hline & & $\begin{array}{l}\text { Pruebas de funcionalidad } \\
\text { implementada. }\end{array}$ & \\
\hline & \multirow{3}{*}{$\begin{array}{l}\text { Visualizar ofertas } \\
\text { de trabajo por } \\
\text { categorías }\end{array}$} & $\begin{array}{l}\text { Diseño de interfaz para visualización } \\
\text { de ofertas de trabajo registradas. }\end{array}$ & \multirow[t]{3}{*}{20} \\
\hline & & $\begin{array}{l}\text { Creación de funcionalidad para } \\
\text { conexión con Firebase y obtención de } \\
\text { ofertas de trabajo. }\end{array}$ & \\
\hline & & $\begin{array}{l}\text { Pruebas de funcionalidad } \\
\text { implementada. }\end{array}$ & \\
\hline
\end{tabular}

Entregable: página administrativa con todos sus módulos terminados. Aplicación móvil con navegabilidad por tipo de usuarios y pantalla para realizar registro de ofertas de trabajo. 


\subsection{Estándares}

Dentro del desarrollo del proyecto se utilizaron los siguientes:

- Codificación: PascalCase, camelCase y UpperSnakeCase en todo el estilo de escritura del proyecto (Martínez, s. f.).

- Lenguajes de programación: Kotlin (Orozco, 2021) en la aplicación móvil y TypeScript (Hernández, 2018) en la página administrativa.

- Librerías de diseño: Material Design (Google, s. f.) en la aplicación móvil y Angular Material librería basada en Material Design para la página administrativa.

- Modelo de base de datos: Firebase (Giraldo, 2021) con un modelo de no relacional basado en JSON (IBM, s. f.).

\section{Resultados}

Como resultados del presente proyecto se pueden ver reflejados en las pruebas realizadas al desarrollo. A continuación, se detalla las pruebas realizadas a la aplicación móvil y la página administrativa que permiten verificar el cumplimiento de las especificaciones planteadas y aprobar su funcionalidad.

En la Tabla 5 se puede ver un ejemplo de una prueba funcional realizada al registro y visualización de ofertas de trabajo.

Tabla 5.

Prueba de aceptación registro y visualización de ofertas de trabajo

\begin{tabular}{lllr}
\hline \multicolumn{1}{c}{ Objetivo } & \multicolumn{1}{c}{ Descripción } & Historias & Estado \\
\hline Validar la & El cliente / solicitante & $3,4,8,9$ & Aprobado \\
publicación de & debe escoger una & & \\
ofertas de trabajo & categoría e ingresar la & & \\
por categoría y su & información que se le & & \\
correspondiente & solicite para registrar & & \\
visualización & $\begin{array}{l}\text { la oferta. El profesional } \\
\text { podrá visualizar la oferta }\end{array}$ & & \\
& y ver el detalle de la & & \\
& oferta \\
\hline
\end{tabular}


En las Figuras 3, 4, 5 se observan las pantallas para iniciar sesión por medio de credenciales o una cuenta de Google; además, la pantalla de registro para los dos tipos de usuarios que utilizan la aplicación.

Figura 3

Pantalla de inicio de sesión
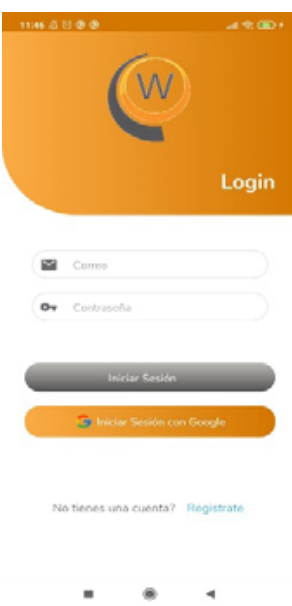

Figura 4

Pantalla de inicio de sesión Google

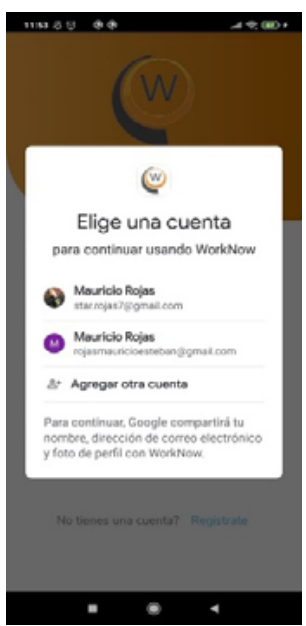

\section{Figura 5}

Pantalla de registro usuario

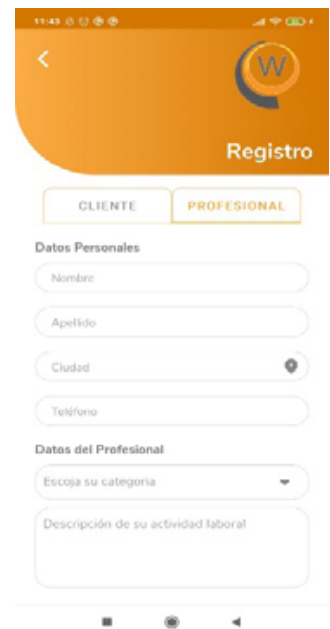

En las Figuras 5, 6, 7 se visualizan las pantallas del registro de solicitud de trabajo, donde el cliente / solicitante llena cada uno de los campos disponibles en el formulario y que servirán para que el profesional acepte o no la solicitud.

\section{Figura 6}

Pantalla de registro de solicitud - formulario
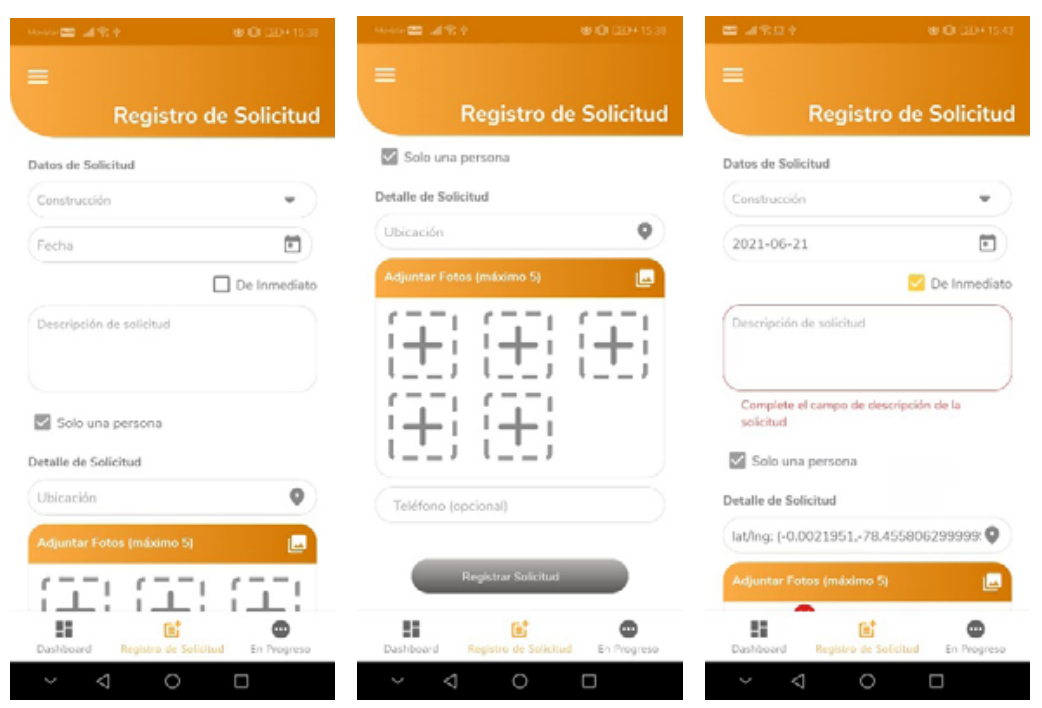

Registro de Solicitud

Detalle de Solkitud
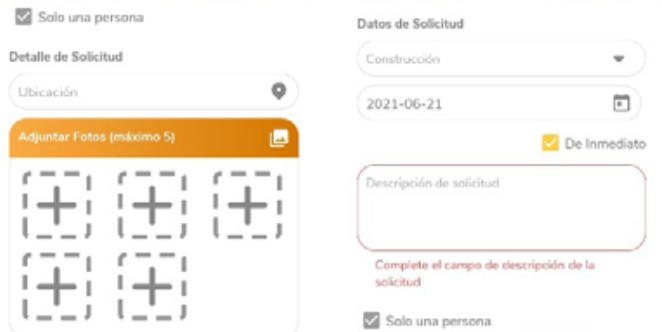

Detalle de Solicitud

lathing: $[-0.0021951 .78,455906299999$ ?
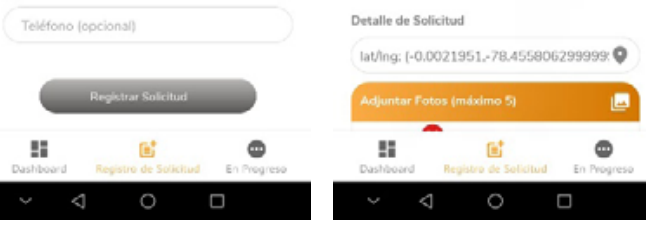

Hif

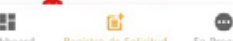

$\checkmark \triangleleft \quad \circ \quad \square$ 


\section{Figura 7}

Pantalla de registro de solicitud - cuadros de diálogo
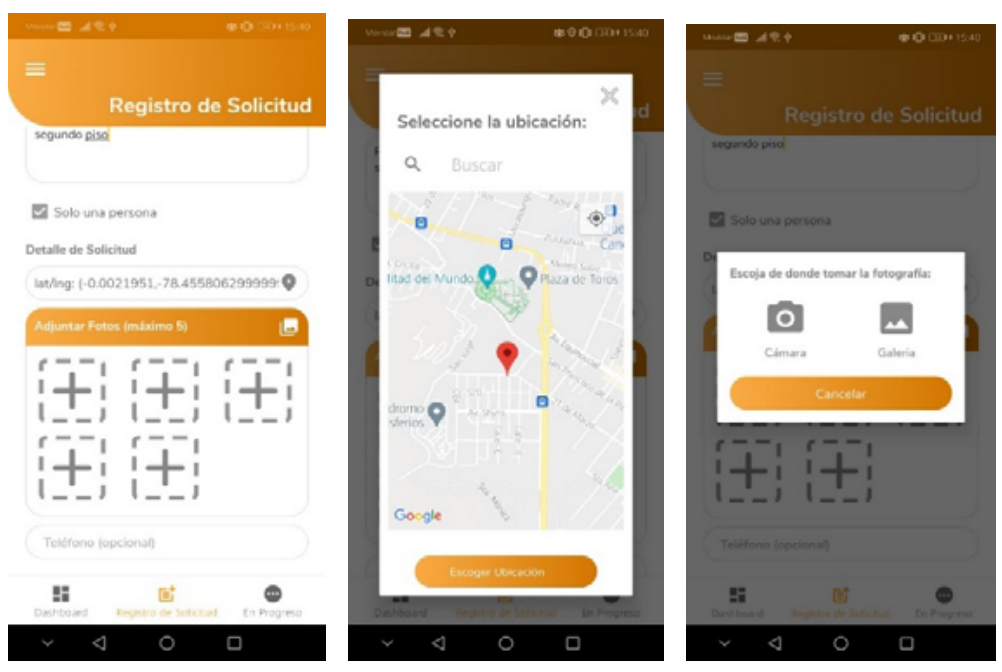

Figura 8:

Pantalla de registro de solicitud - toma de fotografía y visualización
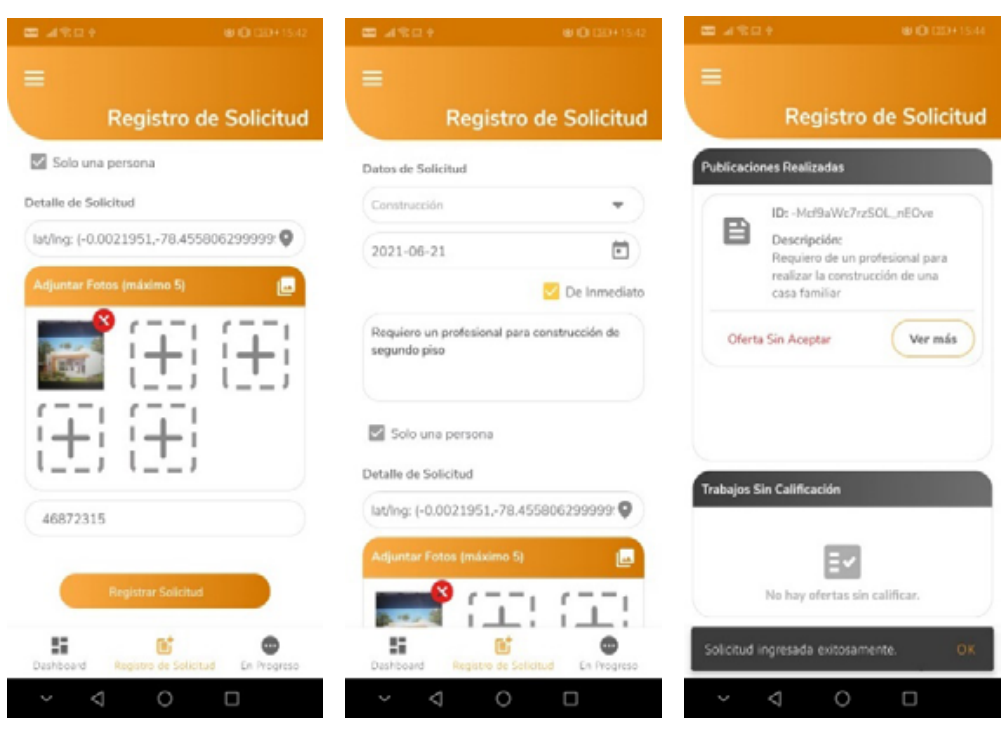

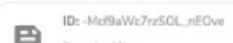

를

Descripcione:

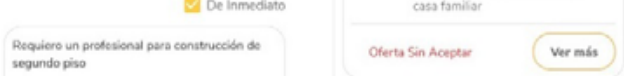

$\nabla$ Solo una persona

Detalle de Solkitud

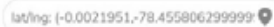

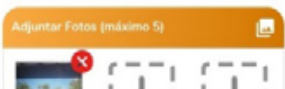

:i i

$\checkmark \triangleleft \quad \circ \quad \square$

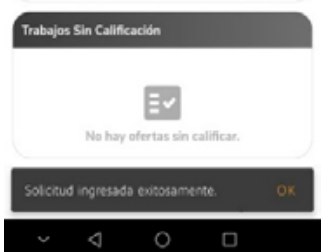


Las Figuras 9, 10, 11 corresponden a la lista de ofertas publicadas y cómo el profesional puede visualizar toda la información registrada por el cliente / solicitante. Una vez que el profesional acepte la oferta al cliente / solicitante le llegará una notificación la cual avisará que su solicitud fue aceptada.

Figura 9

Pantalla de visualización ofertas

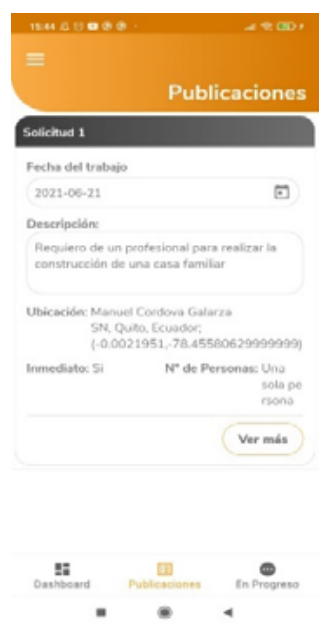

Figura 10

Pantalla de detalle oferta

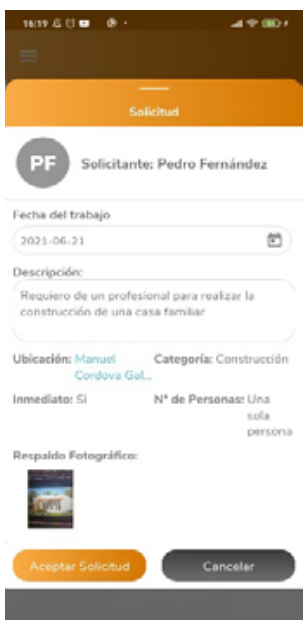

Figura 11

Pantalla de notificación solicitud aceptada

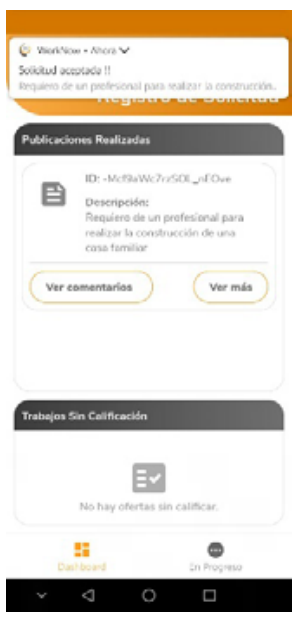

En las Figuras 12 y 13 se puede ver que, al aceptar la solicitud, tanto el cliente /solicitante y el profesional tendrán disponible una interfaz que permitirá la comunicación con el envío de comentarios entre ambos usuarios, lo que facilitará el llegar a un acuerdo por la solicitud aceptada.

Figura 12

Pantalla dashboard con notificación pendiente

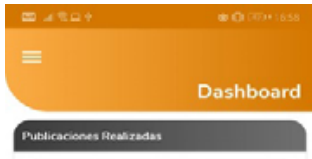

틀

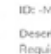

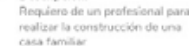
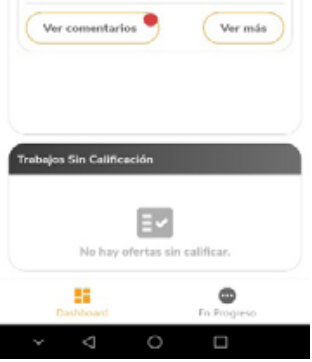

Figura 13

Pantalla de cometarios y notificación

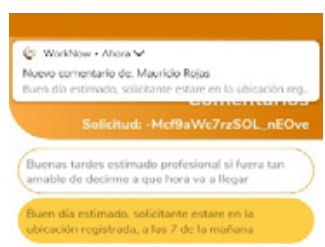


En las Figuras 14, 15 se observan las pantallas finales del proceso de contratación de servicios profesionales, donde se da por terminado el trabajo solicitado previo la validación del cliente / solicitante y se finaliza con la calificación del profesional.

Figura 14

Pantalla en progreso terminar oferta

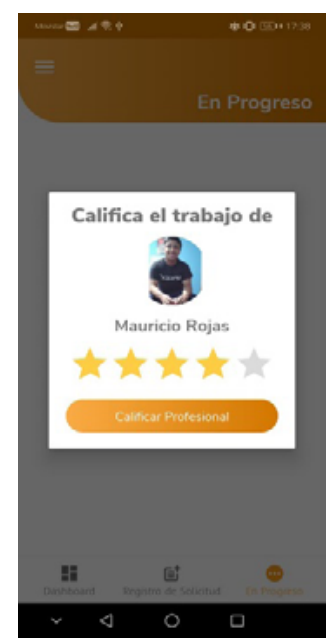

Figura 15

Pantalla calificación profesional

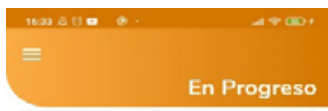

[응

rectia del trabapo

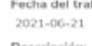

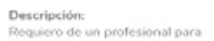

realizar la construcción de una casa

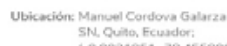

Inmediato: Si

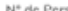
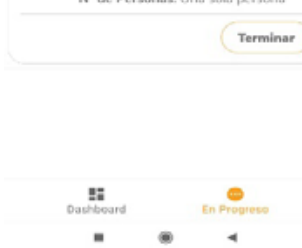

Y en la Figura 16 los usuarios podrán visualizar sus datos personales registrados previamente en la pantalla de registro, en la cual los usuarios podrán actualizar sus datos personales.

Figura 16

Pantalla perfil usuario

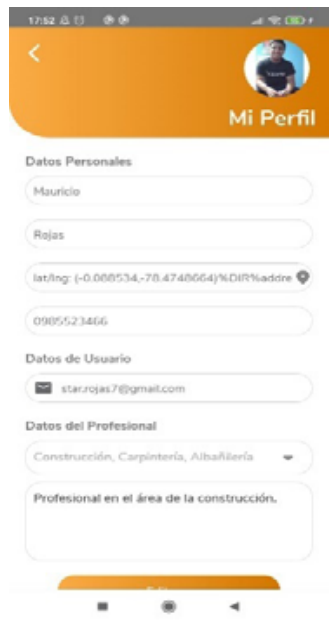


En la Figura 17 se muestra la página de inicio de sesión de uso exclusivo para el usuario administrador, la cual le permitirá acceder a las diferentes páginas que cuenta este sitio web.

\section{Figura 17}

Pantalla de inicio de sesión página administrativa

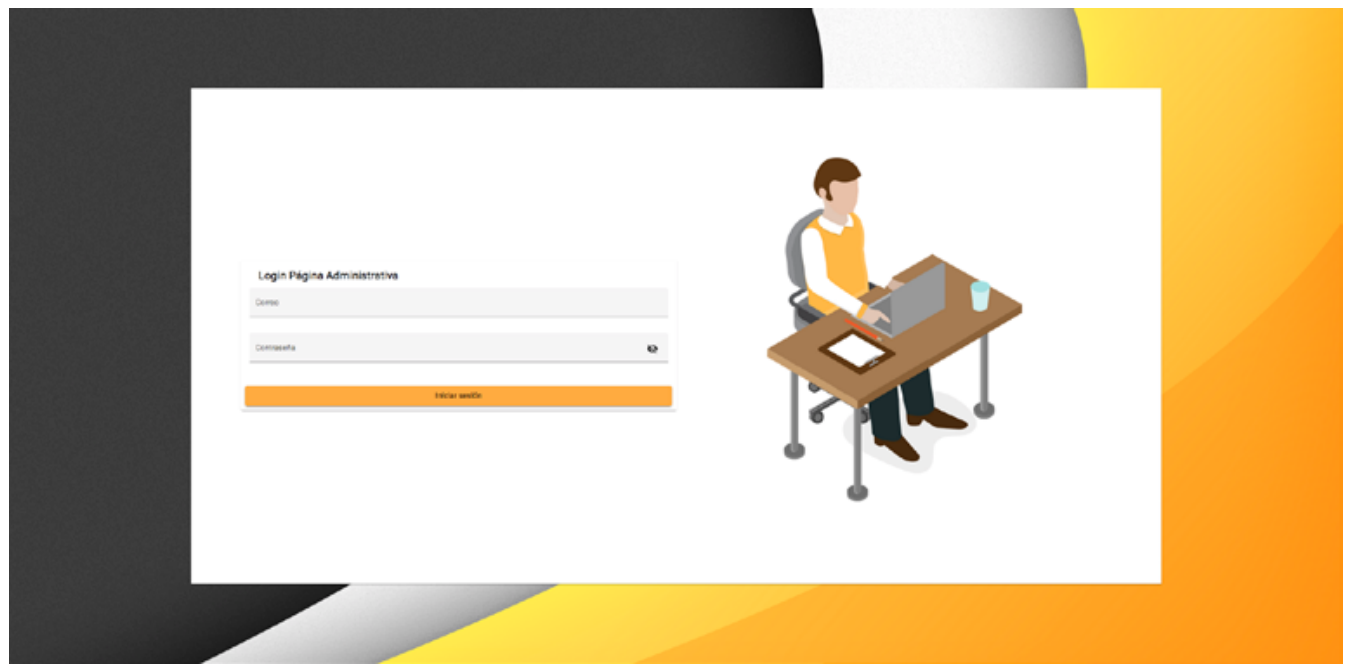

En la Figura 18, al iniciar sesión se muestra la primera página, la cual corresponde a la visualización de las diferentes ofertas de trabajo publicadas por categoría, en esta se podrán ver los datos registrados por los usuarios, así como validar su estado actual.

\section{Figura 18}

Pantalla de ofertas de trabajo registradas

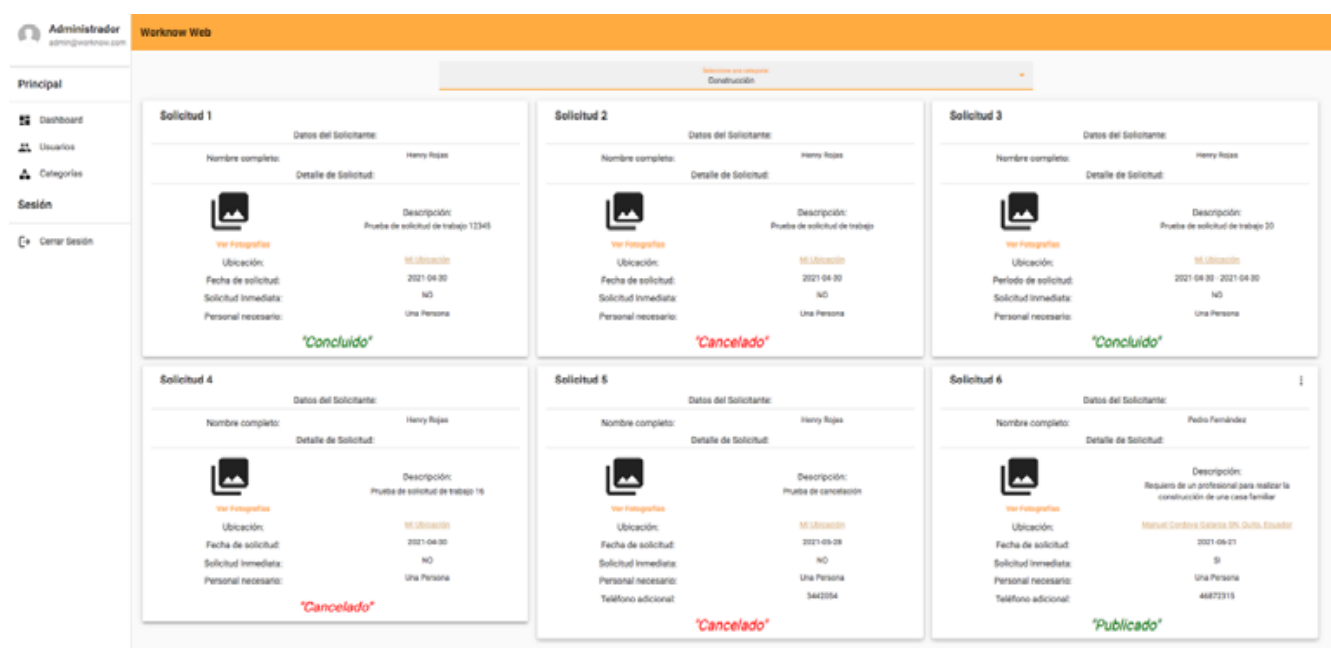


En la Figura 19 se muestra la segunda página, que corresponde a la visualización de los usuarios que utilizan la aplicación móvil, en esta se podrán visualizar los datos personales de los usuarios, así como tener un botón de acción para bloquear al usuario en caso de ser necesario.

Figura 19

Pantalla de usuarios registrados

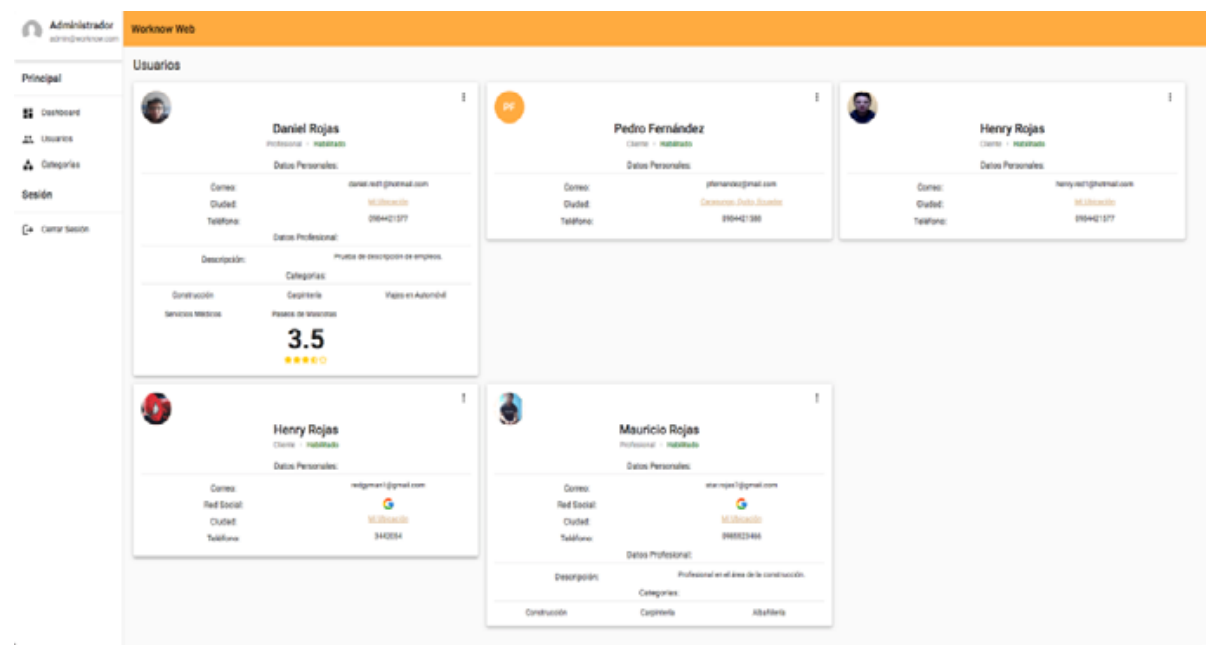

Y finalmente, en la Figura 20 se observa la última página, que pertenece a la administración de categorías, siendo esta la encargada de ingresar, actualizar, deshabilitar y mostrar las categorías que utilizará la aplicación móvil para el registro de solicitudes, así como para la visualización de los campos del formulario.

Figura 20

Pantalla de administración de categorías

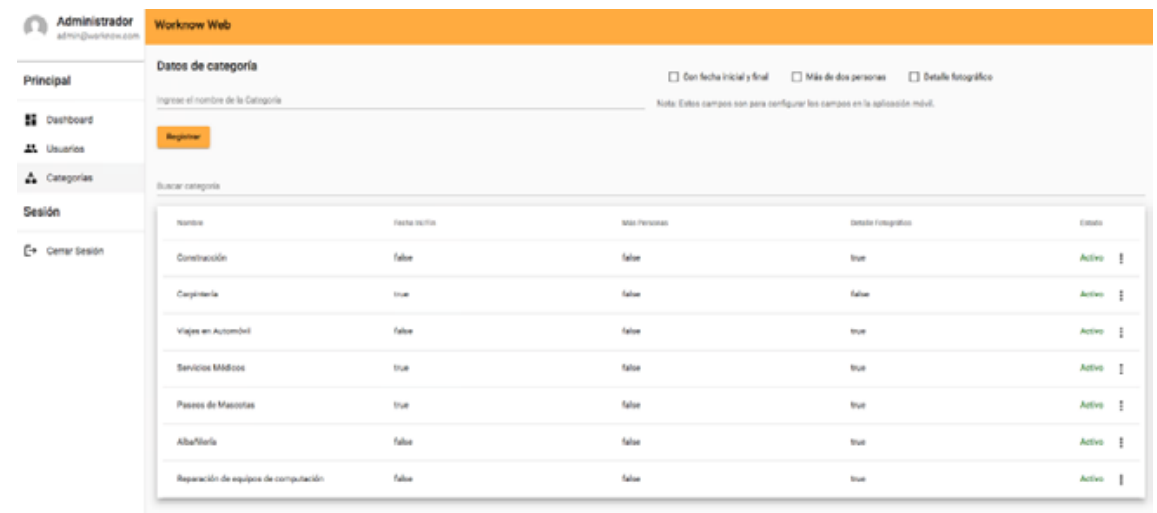




\section{Conclusiones}

El levantamiento de requerimientos fue un factor inicial importante en el desarrollo del proyecto, donde se estableció las funcionalidades principales que tendrá el prototipo para cumplir la implementación del proceso de contratación de servicios profesionales automatizado.

Firebase, como backend del prototipo propuesto, permitió enfocarse principalmente en el desarrollo de la aplicación móvil y la página administrativa, ya que la administración de usuarios y el manejo de la persistencia de información fue exclusivo de la plataforma y evitó la necesidad de generar servicios web para obtener y enviar información.

En el desarrollo de la aplicación móvil se utilizó Android Studio con Kotlin, un lenguaje que permitió reducir el tiempo de desarrollo al realizar una menor codificación. Para la página administrativa, Angular, con su arquitectura de componentes, agilizó la creación de la página web, ya que facilita la reutilización. Estas herramientas permitieron cumplir los objetivos propuestos y bajo la metodología Scrum, los objetivos y tiempos establecidos para este proyecto.

Las pruebas funcionales en la aplicación móvil y página administrativa permitieron verificar su correcto funcionamiento y realizar la corrección de errores vistos durante las pruebas, también comprobar que todos los requisitos establecidos en las historias de usuarios fueron cumplidos. 


\section{Referencias}

Aitana Soluciones ERP y CRM. (16 de octubre de 2018). Visual Studio Code: Funcionalidades y extensiones. El Blog de Aitana. https://blog.aitana.es/2018/10/16/visual-studio-code/

Cabify. (s. f.). Cabify. https://cabify.com/ec

CompuTrabajo. (s. f.). CompuTrabajo. https://www.computrabajo.com.ec/

Deemer, P., Benefield, G., Larman, C., \& Vodde, B. (2009). Información básica de SCRUM. Scrum Training Institute. http://libroslibres.uls.edu.sv/informatica/informacion basica scrum.pdf

Developers. (17 de mayo de 2021). Introducción a Android Studio. Android Developers. https://developer.android.com/studio/intro?hl=es-419

Giraldo, V. (16 de abril de 2019). ¿Ya conoces Firebase? La herramienta de desarrollo y análisis de aplicaciones mobile. Rock Content https://rockcontent.com/es/blog/que-es-firebase/

Google. (s. f.). Material Design. Material.io. https://material.io/design

Grijalva, A.M. (21 de agosto de 2020). Jóvenes en la informalidad y lo que sabemos sobre sus condiciones de trabajo: Parte 1, levantamiento de información. PNUD Ecuador. https://www.ec.undp.org/content/ecuador/ es/home/blog/2020/jovenes-en-la-informalidad-y-lo-que-sabemos-sobre-sus-condicione.html

Hernández, U. (03 de junio de 2018). Qué es TypeScript. CódigoFacilito. https://codigofacilito.com/articulos/ typescript

IBM. (s. f.). Formato JSON (JavaScript Object Notation). https://www.ibm.com/docs/es/baw/20.x?topic=formats-javascript-object-notation-json-format

inDriver. (s. f.). inDriver. https://indriver.com/es/city/

Lozano Chaguay, L., Lozano Chaguay, S., \& Robledo Galeas, R. (2020). Desempleo en tiempos de Covid-19: efectos socioeconómicos en el entorno familiar. Journal of Science and Research: Revista Ciencia e Investigación, 5(4), 187-197. https://revistas.utb.edu.ec/index.php/sr/article/view/926/667

Martínez, A. B. (s. f.). Nomenclaturas de programación: camelCase, PascalCase, snake_case. Platzi. https:// platzi.com/clases/2218-pensamiento-logico/35651-nomenclaturas-de-programacion-camelcase-pascalcase/

Multitrabajos. (s. f.). multitrabajos. https://www.multitrabajos.com/ 
OIT. (24 de febrero de 2021). Plataformas digitales: un futuro del trabajo con oportunidades y desafíos para América Latina y el Caribe. Organización Internacional del Trabajo. https://www.ilo.org/americas/sala-de-prensa/WCMS 773309/lang--es/index.htm

Opcionempleo. (s. f.). Opciónempleo. https://www.opcionempleo.ec/

Orozco, A. G. (05 de abril de 2021). Qué es Kotlin y características. OpenWebinars https://openwebinars.net/ blog/que-es-kotlin/

Uber. (s. f.). Uber. https://www.uber.com/global/es/sign-in/ 
Copyright (c) 2021 Henry Daniel Rojas Cisneros

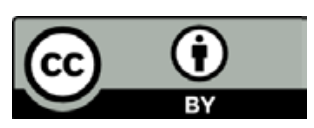

Este texto está protegido bajo una licencia internacional Creative Commons 4.0.

Usted es libre para Compartir-copiar y redistribuir el material en cualquier medio o formato - y Adaptar el documento - remezclar, transformar y crear a partir del material-para cualquier propósito, incluso para fines comerciales, siempre que cumpla las condiciones de Atribución. Usted debe dar crédito a la obra original de manera adecuada, proporcionar un enlace a la licencia, e indicar si se han realizado cambios. Puede hacerlo en cualquier forma razonable, pero no de forma tal que sugiera que tiene el apoyo del licenciante o lo recibe por el uso que hace de la obra.

Resumen de licencia - Texto completo de la licencia 